\title{
(2) OPEN ACCESS \\ Evaluation of intravitreal topotecan dose levels, toxicity and efficacy for retinoblastoma vitreous seeds: a preclinical and clinical study
}

\author{
Carley M Bogan, ${ }^{1}$ Jessica V Kaczmarek, ${ }^{1}$ Janene M Pierce, ${ }^{1}$ Sheau-chiann Chen, ${ }^{2,3}$ \\ Kelli L Boyd ${ }^{3,4}$ Marion W Calcutt, ${ }^{5}$ Thomas M Bridges, ${ }^{6}$ Craig W Lindsley, ${ }^{6}$ \\ Jennifer B Nadelmann (1) , ${ }^{7}$ Albert Liao, ${ }^{7}$ Terry Hsieh, ${ }^{7}$ David H Abramson, ${ }^{7}$ \\ Jasmine H Francis (1) , ' Debra L Friedman, ${ }^{3,8}$ Ann Richmond, $3,9,10,11$ \\ Anthony B Daniels (1) 1,3,11,12
}

For numbered affiliations see end of article.

\section{Correspondence to} Dr Anthony B Daniels, Vanderbilt Eye Institute, 2311 Pierce Avenue, Vanderbilt University Medical Center, Nashville, Tennessee, USA; anthony.b.daniels@vumc.org

Received 3 December 2020 Revised 9 April 2021 Accepted 13 April 2021

Check for updates

(C) Author(s) (or their employer(s)) 2021. Re-use permitted under CC BY-NC. No commercial re-use. See rights and permissions. Published by BMJ.

\section{To cite: Bogan CM,} Kaczmarek JV, Pierce JM et al. Br J Ophthalmol Epub ahead of print: [please include Day Month Year]. doi:10.1136/

bjophthalmol-2020-318529

\section{ABSTRACT}

Background Current melphalan-based intravitreal regimens for retinoblastoma (RB) vitreous seeds cause retinal toxicity. We assessed the efficacy and toxicity of topotecan monotherapy compared with melphalan in our rabbit model and patient cohort.

Methods Rabbit experiments: empiric pharmacokinetics were determined following topotecan injection. For topotecan $(15 \mu \mathrm{g}$ or $30 \mu \mathrm{g})$, melphalan $(12.5 \mu \mathrm{g})$ or saline, toxicity was evaluated by serial electroretinography (ERG) and histopathology, and efficacy against vitreous seed xenografts was measured by tumour cell reduction and apoptosis induction. Patients: retrospective cohort study of 235 patients receiving 990 intravitreal injections of topotecan or melphalan.

Results Intravitreal topotecan $30 \mu \mathrm{g}$ (equals $60 \mu \mathrm{g}$ in humans) achieved the $I C_{90}$ across the rabbit vitreous. Three weekly topotecan injections (either $15 \mu \mathrm{g}$ or $30 \mu \mathrm{g}$ ) caused no retinal toxicity in rabbits, whereas melphalan $12.5 \mu \mathrm{g}$ (equals $25 \mu \mathrm{g}$ in humans) reduced ERG amplitudes $42 \%-79 \%$. Intravitreal topotecan $15 \mu \mathrm{g}$ was equally effective to melphalan to treat WERI-Rb1 cell xenografts in rabbits $(96 \%$ reduction for topotecan vs saline ( $p=0.004), 88 \%$ reduction for melphalan vs saline ( $p=0.004)$, topotecan vs melphalan, $p=0.15)$. In our clinical study, patients received 881 monotherapy injections (48 topotecan, 833 melphalan). Patients receiving $20 \mu \mathrm{g}$ or $30 \mu \mathrm{g}$ topotecan demonstrated no significant ERG reductions; melphalan caused ERG reductions of $7.6 \mu \mathrm{V}$ for every injection of $25 \mu \mathrm{g}$ $(p=0.03)$ or $30 \mu g(p<0.001)$. Most patients treated with intravitreal topotecan also received intravitreal melphalan at some point during their treatment course. Among those eyes treated exclusively with topotecan monotherapy, all eyes were salvaged.

Conclusions Taken together, these experiments suggest that intravitreal topotecan monotherapy for the treatment of RB vitreous seeds is non-toxic and effective.

\section{INTRODUCTION}

Vitreous seeds have historically been the most difficult-to-treat aspect of intraocular retinoblastoma (RB). ${ }^{1}{ }^{2} \mathrm{RB}$ tumours with vitreous seeds are those least likely to be salvaged with radiation $^{3}$ or intravenous chemotherapy. ${ }^{1245}$ Newer approaches to delivering chemotherapy, including intra-arterial chemotherapy (IAC) ${ }^{6}$ and direct intravitreal injection of chemotherapy, ${ }^{78}$ have partially overcome the treatment-resistant nature of vitreous seeds and have improved globe salvage rates for RB. ${ }^{10}$ However, the primary chemotherapeutic agent used in both IAC and intravitreal chemotherapy is melphalan, which has been associated with retinal toxicity. ${ }^{811-13}$ Thus, while intravitreal melphalan may be effective, retinal functional loss is common. ${ }^{12}{ }^{14}$ Furthermore, the toxicity is dosedependent ${ }^{15}$ and worsens with each subsequent melphalan injection delivered. ${ }^{12} 1316$

Recently, topotecan has been explored as an alternative chemotherapy agent, both by intravenous, ${ }^{17}$ intra-arterial ${ }^{18}$ and intravitreal ${ }^{19} 20$ routes. Preliminary laboratory and clinical evidence suggests that topotecan may be less toxic than current standard-of-care melphalan. ${ }^{20}{ }^{21}$ However, it is unclear if a non-toxic dose of topotecan is clinically effective. ${ }^{21}$ Further, it is unclear just how effective topotecan is as monotherapy, as many centres have generally used it in combination with melphalan, ${ }^{22}$ or have been quick to re-add melphalan back into the regimen if topotecan monotherapy appeared to not achieve adequate tumour control. ${ }^{20}$ Likewise, the optimal dose of topotecan that best balances efficacy with toxicity as intravitreal monotherapy has not been established.

We recently developed a rabbit xenograft model of RB with vitreous seeds and retinal tumours, which we have used to study the toxicity of IAC, exploring various different IAC drugs. ${ }^{23}{ }^{24}$ We have also previously described a complete platform to assess functional and structural retinal toxicity associated with local delivery of various chemotherapeutic agents. ${ }^{11}$ Here, we use this rabbit model ${ }^{23}$ and this toxicity evaluation platform, ${ }^{11}$ to determine the dose of intravitreal topotecan which is effective and non-toxic when delivered as monotherapy. We then corroborate this evidence of non-toxicity with our clinical experience treating RB patients with vitreous seeds with intravitreal topotecan.

\section{METHODS}

\section{Statement of research ethics}

All animal experiments adhered to the Association for Research in Vision and Ophthalmology 
Statement on Animal Use and were performed under the auspices of the Vanderbilt Institutional Animal Care and Use Committee.

\section{Intravitreal topotecan pharmacokinetics}

New Zealand white rabbits $(2.8-3.0 \mathrm{~kg})$ were used for all studies. For pharmacokinetic experiments, a 20 -gauge valved vitrectomy cannula was inserted $2-3 \mathrm{~mm}$ behind the limbus. One microgram topotecan hydrochloride was injected on the opposite side $2-3 \mathrm{~mm}$ behind the limbus into the vitreous cavity. Serial vitreous taps were performed through the valved cannula at $30 \mathrm{~min}, 1$ hour, 2 hours, 4 hours and 6 hours. Use of a valved cannula-maintained eye stability and prevented efflux of vitreous contents during manipulations. ${ }^{25}$ Vitreous samples were immediately placed on dry ice and then stored at $-80^{\circ} \mathrm{C}$ until drug levels were measured.

Vitreous samples were thawed, an internal carbamazepine standard was added and samples were diluted with blank plasma, then deproteinised with acetonitrile. Samples were analysed on a Thermo Scientific TSQ Quantum Ultra mass spectrometer interfaced to a Waters Acquity UPLC system, using methodology we have reported previously. ${ }^{23}$

Topotecan concentrations were averaged across rabbits at each time point. The resulting mean time-concentration data from each matrix were analysed via non-compartmental analysis (Phoenix WinNonlin V.6.4, Pharsight/Certara USA, Princeton, New Jersey, USA) to determine pharmacokinetic parameters, including half-life.

\section{In vitro determination of dosing}

Human WERI-Rb1 RB cells $\left(5 \times 10^{3}\right)$ were plated in 96-well plates in the presence of various concentrations of topotecan for 16 hours (five half-lives as determined through the above pharmacokinetic experiments). Topotecan-containing media was then removed, and fresh media added. After 7 days, the CellTiter Blue assay (Promega, Madison, Wisconsin, USA) was used to count live cells. Survival curves were graphed with GraphPad, and the $\mathrm{IC}_{90}$ was calculated.

Using the pharmacokinetic parameters determined above, we calculated $^{25}$ the dose of topotecan that would need to be injected into the eye to achieve the $\mathrm{IC}_{90}$ in the vitreous on the opposite side of the eye for a duration of five half-lives.

\section{Assessment of efficacy of intravitreal topotecan for vitreous seeds in rabbits}

Figure 1A depicts our experimental design. RB vitreous seeds were created by intravitreal injection of 1000000 WERI-Rb1 cells in
$100 \mu \mathrm{L}$ saline in both eyes of cyclosporine-immunosuppressed rabbits, as we have described previously. ${ }^{23}{ }^{25}$ After 2 weeks of growth, the right eyes received three weekly injections of either $15 \mu \mathrm{g} / 100 \mu \mathrm{L}$ topotecan or $12.5 \mu \mathrm{g} / 100 \mu \mathrm{L}$ melphalan, while all left eyes received $100 \mu \mathrm{L}$ saline.

Two weeks after the final injection, all rabbits were euthanised, and the eyes were removed. For five rabbits, the vitreous of each eye was harvested and digested in $0.5 \mathrm{mg} / \mathrm{mL}$ hyaluronidase and $1 \mathrm{mg} / \mathrm{mL}$ collagenase overnight at $37^{\circ} \mathrm{C}$. Live cells were counted by direct microscopy using trypan blue stain. In four additional rabbits from each treatment group, the entire eyes were submitted for histopathology (two rabbits after receiving three injections and two rabbits after receiving a single injection).

\section{Assessment of ocular toxicity of intravitreal topotecan in rabbits}

Four cohorts ( $n=4-6$ rabbits/cohort) received either topotecan $30 \mu \mathrm{g}$ (the calculated $\mathrm{IC}_{90}$ ), topotecan $15 \mu \mathrm{g}$ (half the calculated $\mathrm{IC}_{90}$ ), saline (control) or melphalan $12.5 \mu \mathrm{g}$ (current standardof-care) ${ }^{26}$ In all rabbits within a given cohort, the right eyes received three injections, one injection per week, of the same drug/dose. Figure 1B depicts our experimental design. Electroretinography (ERG; OcuScience, Henderson, Nevada, USA) was performed according to the modified International Standard for Clinical Electrophysiology of Vision protocol for rabbits. ${ }^{27}$ Intravitreal injections were performed weekly, and always within 1 day following testing. After euthanasia, eyes were harvested and fixed in Davidson's solution.

Toxicity was defined for every ERG parameter, using our previously published definition. ${ }^{11}{ }^{25}$ Briefly, toxicity was deemed significant for a given dose in a rabbit group if there was a $25 \%$ reduction in average ERG amplitude, or a $25 \%$ prolongation of average implicit time comparing the post-treatment parameter values after three injections with the pretreatment values, if the difference was statistically significant.

\section{Ocular toxicity and efficacy of intravitreal topotecan versus melphalan in patients}

Medical records of all patients treated with intravitreal injections at Memorial Sloan-Kettering Cancer Center and Vanderbilt University Medical Center were reviewed. Patients receiving intravitreal topotecan were identified. A second cohort of all patients receiving melphalan as monotherapy were included as a comparator group. Injection number, drug and dose were recorded. ERGs were performed using a previously published,
A

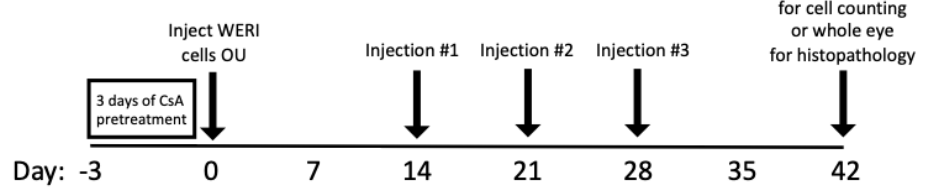

B

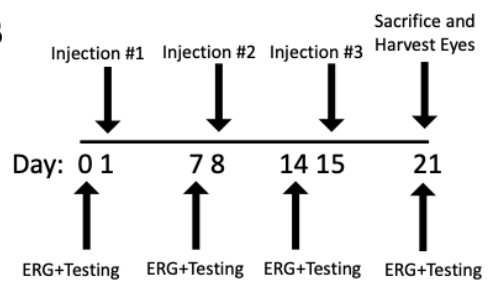

Figure 1 Experimental design of efficacy and toxicity experiments in the rabbit model. (A) Efficacy experiments. Following 3 days of cyclosporine immunosuppression (which also continued throughout the course of the experiment), WERI-Rb1 cells were injected into the vitreous of both eyes of New Zealand white rabbits. After 2 weeks of growth, once-per-week injections of drug (topotecan $15 \mu \mathrm{g}$ or melphalan $12.5 \mu \mathrm{g}$ ) were given into the right eye (OD), and saline into the left eye (OS). Imaging was obtained and rabbits were sacrificed 2 weeks after the third injection. (B) Toxicity experiments. Drug (topotecan, melphalan or saline, depending on the cohort) was injected into the right eye (OD) of New Zealand white rabbits once per week for three consecutive weeks. One day prior to each injection, functional testing was obtained (see the Methods section). One week following the final (third) injection, testing was again performed, and then the rabbits were sacrificed and globes harvested for histopathological evaluation. ERG, electroretinography. CsA=cyclosporine $A$. 
and validated, abbreviated ERG protocol. ${ }^{28-30}$ For efficacy, we included all treated patients for which complete records were available. Our toxicity analyses included only those monotherapy injections for which this ERG protocol was performed prior to the intravitreal injection, as well as subsequent to the injection, and patients who also received concomitant IAC between the two ERGs were excluded. Ocular and systemic adverse events, as well as clinical outcomes, were recorded.

\section{Statistical analyses of rabbit and human efficacy and ERG data}

For univariate analysis to compare toxicity in patients, the Wilcoxon signed-rank test was used. For multivariable analysis, to evaluate the toxicity of each drug at different dosages, a linear mixed-effects model was fitted with treatment groups and the repeated measurements (pre or post) for each parameter and each test. Using model-based (least-square) means, the average adjusted change from pretreatment versus post-treatment and the difference in change between different treatment groups (difference-of-differences) were estimated and compared with the Wald test. Our predefined definition of toxicity (see earlier) was used in the rabbit analyses. Data were transformed to better meet normality assumptions and adjusted for heteroscedasticity when necessary. To account for multiple comparisons, Bonferroni-adjusted $\mathrm{p}$ values were reported (two-tailed), with adjusted $\mathrm{p}$ values less than 0.05 considered statistically significant. The analyses were performed using R V.3.6.3 including packages 'nlme' and 'emmeans'. For experiments in rabbits, 'pre' and 'post' were defined before and after the three injections. For human experiments, because of the variability between dosing and interval of injections, 'pre' and 'post' were defined on a 'per injection' basis without minimum reduction limits, and inter-eye/ intra-patient and intra-eye correlations were taken into account in our modelling.

For efficacy experiments in rabbits comparing paired right eyes receiving topotecan (or melphalan) and contralateral left eyes receiving saline, the paired t-test was used. Relative reduction of cell counts was analysed to compare the difference between two independents groups (topotecan and melphalan rabbit cohorts) using Welch two sample t-test.

A

Pharmacokinetics of Intravitreal Topotecan

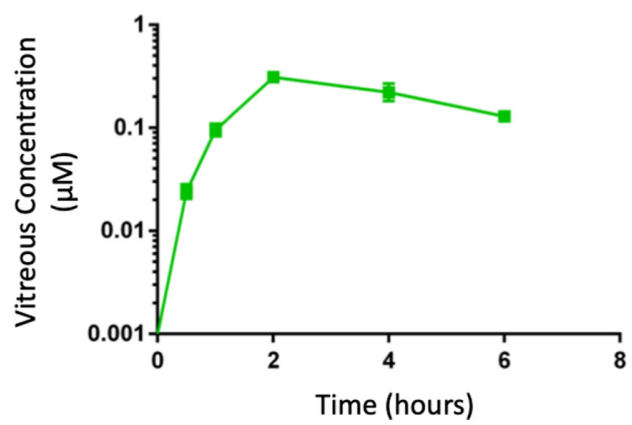

RESULTS

In vivo topotecan pharmacokinetics and in vitro determination of expected effective in vivo dose

Peak concentration at the opposite side of the eye was achieved at 2 hours postinjection (figure $2 \mathrm{~A}$ ). The average $\mathrm{C}_{\max }$ at the opposite side of the eye was $0.31 \mu \mathrm{mol} / \mathrm{L}$. However, the theoretical $\mathrm{C}_{\max }$ (calculated as $1 \mu \mathrm{g} / 1.4 \mathrm{~mL}$ rabbit vitreous volume) is $1.56 \mu \mathrm{mol} / \mathrm{L}$. Therefore, compared with the theoretical $\mathrm{C}_{\max }$, the actual empiric $\mathrm{C}_{\max }$ at the opposite side of the eye achieved 2 hours after injection was $\sim 20 \%$ of expected, likely due to rapid efflux during this period of slow diffusion of topotecan across the vitreous. The half-life of topotecan in the rabbit eye was 3.27 hours. We therefore exposed WERI-Rb1 human RB cells to various doses of topotecan for five vitreous half-lives $(\sim 16$ hours total $)$ and measured live cells 7 days later. The $\mathrm{IC}_{90}$ was $300 \mathrm{nM}$ (figure 2B). We calculated that we would need to inject $30 \mu \mathrm{g}$ topotecan to sustain this $\mathrm{IC}_{90}$ concentration for 16 hours (five half-lives) at the opposite side of the rabbit eye.

\section{Relative efficacy of intravitreal topotecan versus melphalan to treat RB vitreous seeds in vivo in rabbits}

Three weekly injections of $15 \mu \mathrm{g}$ topotecan killed $96 \%$ of vitreous seed tumour cells, compared with saline-treated contralateral eyes $(p=0.004$, figure 3$)$. Three weekly injections of $12.5 \mu \mathrm{g}$ melphalan (corresponding to the clinically used dose of $25 \mu \mathrm{g}$ in patients) killed $88 \%$ of cells, compared with salinetreated contralateral eyes $(p=0.004$; topotecan vs melphalan: $\mathrm{p}=0.15$, figure 3 ). For additional rabbits in each cohort, the entire eyes were harvested and submitted for histopathology. Residual RB cells in the topotecan-treated eyes were TUNEL positive, suggesting that the $\sim 4 \%$ of 'remaining' cells counted in the vitreous seed quantitation assay were likely in the process of dying as well.

\section{Toxicity of various doses of intravitreal topotecan compared with melphalan in rabbits}

While there was no worsening of ERG parameters in the saline control group, melphalan caused significant worsening of almost all ERG parameters, with reductions in ERG amplitudes between $42 \%$ and $79 \%$ (figure $4 \mathrm{~A}$ ). These ERG changes

B

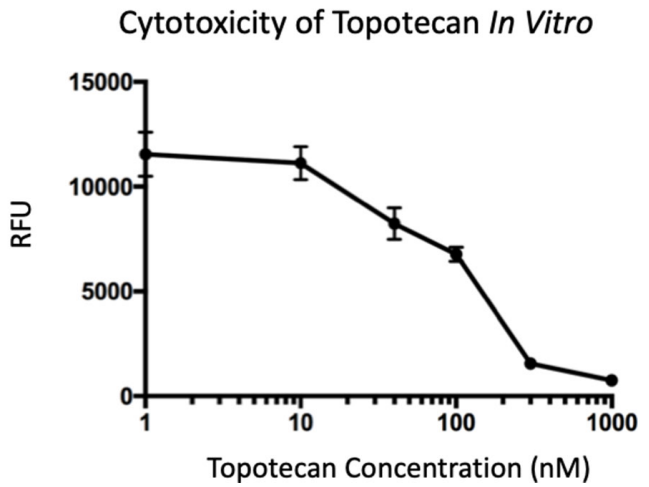

Figure 2 Pharmacokinetics of intravitreal topotecan in the rabbit eye and dose-dependent survival curves of retinoblastoma cells to transient exposure to topotecan in vitro. (A) Pharmacokinetic curve in the rabbit vitreous following intravitreal injection of $1 \mu \mathrm{g}$ of topotecan hydrochloride. Sampling was performed through a valved vitrectomy cannula inserted on the contralateral side of the eye relative to the injection site, and all sampling was performed on the far side of the vitreous relative to the injection site. The use of a valved vitrectomy cannula helped to maintain eye stability and prevent leakage throughout the experiment. (B) CellTiter Blue survival curve in human WERI-Rb1 cells exposed in vitro to various doses of topotecan hydrochloride for 16 hours (equal to five vitreous half-lives). Surviving cells were measured at the 7 -day time-point. The $I C_{90}$ can be found to correspond to $300 \mathrm{nM}$ topotecan hydrochloride for this length of exposure. RFU=relative fluorescence units. 

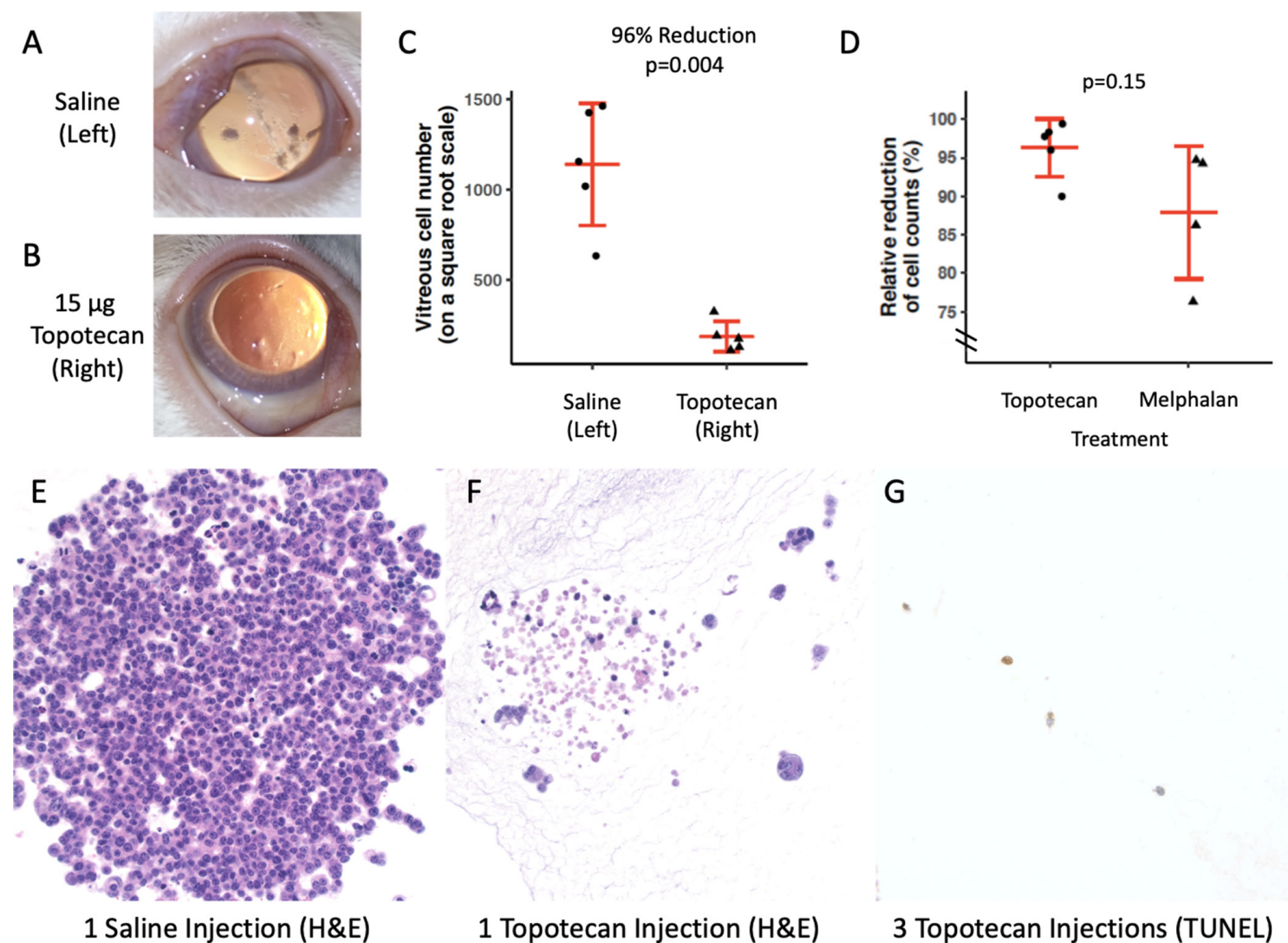

1 Topotecan Injection (H\&E) 3 Topotecan Injections (TUNEL)

Figure 3 Efficacy of intravitreal topotecan against retinoblastoma vitreous seeds in a rabbit xenograft model. One million WERI-Rb1 human retinoblastoma cells were injected into the vitreous of both eyes of cyclosporine-immunosuppressed rabbits. Following 2 weeks of growth, the eyes were given three weekly injections of the intravitreal drug (right eye) or intravitreal saline (left eye). (A-B) Residual vitreous seeds 2 weeks following the final (third) injection, showing (A) significant large seeds in a representative left eye, and (B) complete clinical resolution of the vitreous seeds in a representative topotecan-treated right eye. (C) Quantification of residual live vitreous seeds 2 weeks after the third injection of topotecan (right eyes) or saline (left eyes), showing reduction in live tumour cells with topotecan $15 \mu \mathrm{g}$. Note the scale is a square root scale and is not linear. (D) Comparison of relative reduction with topotecan versus melphalan (compared with their respective saline-treated contralateral eyes), demonstrating no statistically significant difference between the two treatments. Note the abbreviated Y-axis only extends from $75 \%$ to $100 \%$. (E) H\&E stain of a vitreous seed 2 weeks after a single saline injection in the control eye. (F) H\&E stain of a vitreous seed 2 weeks after one single injection of $15 \mu \mathrm{g}$ topotecan, showing significant cell death in most cells. (G) An eye treated with 3 weekly injections of $15 \mu$ topotecan. TUNEL staining shows that all remaining cells are in the process of dying. Taken together with our quantitative cell counting experiments, which showed a $96 \%$ reduction in tumour cells with three weekly injections of topotecan, this indicates that even the 'surviving' $4 \%$ of cells were actually in the process of dying as well. $\mathrm{H} \& \mathrm{E}=$ hematoxylin and eosin.

occurred in every rabbit within the cohort, with a median of 9 (IQR: 7-9, out of 18) parameters affected per rabbit. Similarly, implicit times were prolonged. Histopathology demonstrated severe atrophy affecting all retinal layers, worst near the injection sites (figure 4B-D).

In contrast, rabbits in the $15 \mu \mathrm{g}$ and $30 \mu \mathrm{g}$ topotecan cohorts did not experience any statistically or clinically meaningful worsening of ERG parameters (figure 4A). Even at twice the clinically effective dose, multiple repeated weekly intravitreal topotecan injections did not cause retinal toxicity. No other signs of toxicity were observed on clinical examination, and histopathology showed none of the retinal damage that was seen in the melphalan-treated groups, with retinas of eyes treated with topotecan being histologically indistinguishable from salinetreated control eyes (figure 4B-F).

\section{Comparative toxicity in patients receiving intravitreal topotecan at various doses compared with melphalan} In 41 patients, 108 intravitreal injections of topotecan were given to 42 eyes. Of these 108 injections, 48 injections consisted of topotecan as intravitreal monotherapy, at dosages of either $30 \mu \mathrm{g}$ (18 injections), $20 \mu \mathrm{g}$ (29 injections) or $10 \mu \mathrm{g}$ (one injection). In general, the lower dose of $20 \mu \mathrm{g}$ was used until $\sim 2017$, and $30 \mu \mathrm{g}$ was used beginning in mid-2017, when it was felt that the efficacy-versus-toxicity balance warranted an increase in dose (the single treatment with $10 \mu \mathrm{g}$ was given in 2014). Preinjection and postinjection ERG data were available for 40 topotecan injections. Nine 'undetectable' pretreatment ERGs $(<5 \mu \mathrm{V})$ were excluded. Six additional injections were excluded because they were still receiving concomitant IAC. Ultimately, this left 25 topotecan monotherapy injections (from 14 eyes) with evaluable ERGs for analysis (11 at $20 \mu \mathrm{g}$ and 14 at $30 \mu \mathrm{g}$ ).

In the comparator group, 882 intravitreal melphalan injections were given to 210 eyes of 194 patients. Of these, 833 injections (205 eyes) consisted of melphalan as intravitreal monotherapy (99 injections at $25 \mu \mathrm{g}, 732$ injections at $30 \mu \mathrm{g}, 2$ injections at $40 \mu \mathrm{g})$. Preinjection and postinjection ERG data using the previously described and validated ${ }^{28-30}$ abbreviated clinical protocol were available for 384 injections. Injections were excluded if they were still receiving concomitant IAC, if pretreatment ERGs 


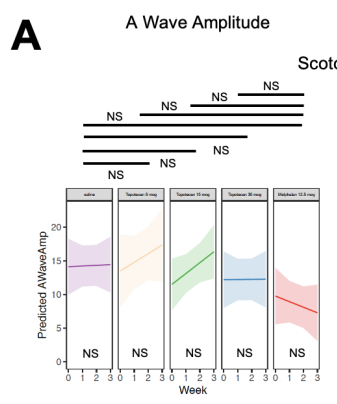

B Wave Amplitude

Scotopic 100
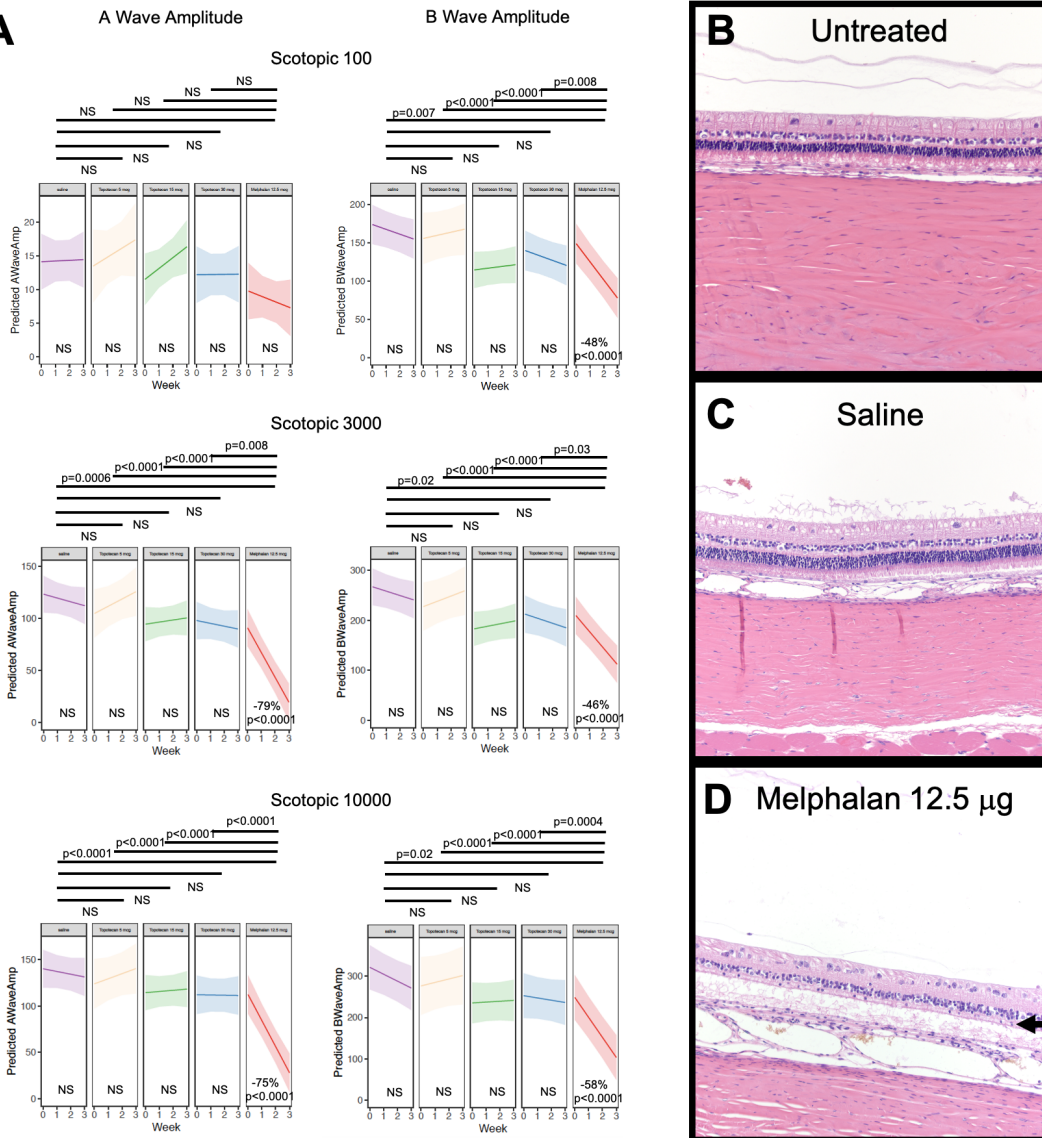

cotopic 10000
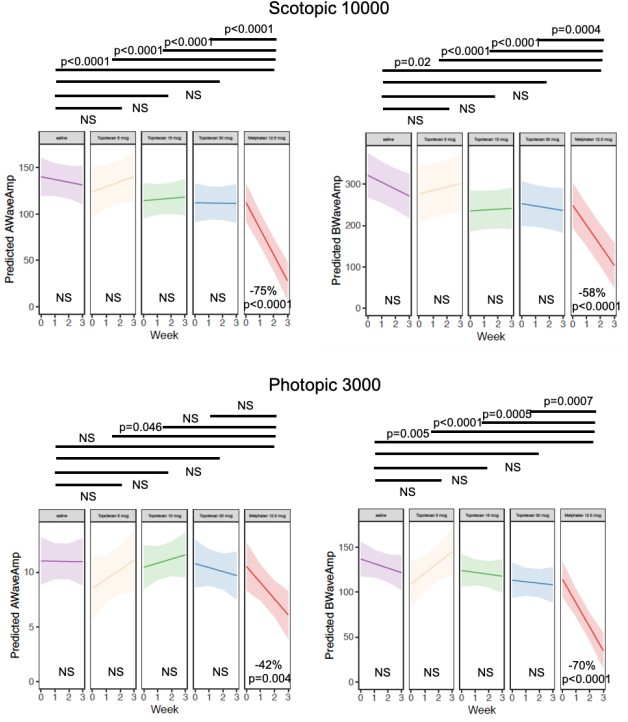

Photopic 3000
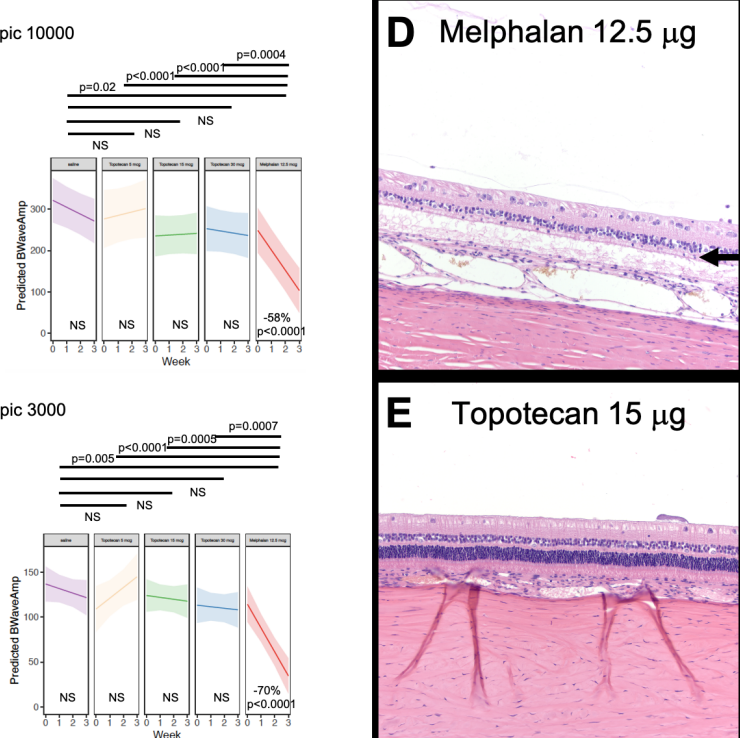

E Topotecan $15 \mu \mathrm{g}$

$30 \mathrm{~Hz}$ Flicker
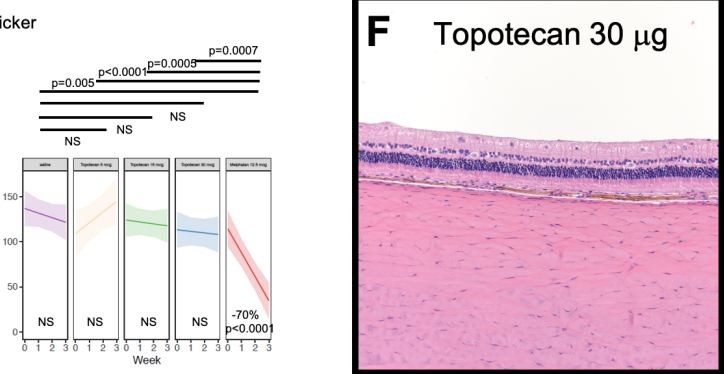

Figure 4 Absence of retinal toxicity with various doses of intravitreal topotecan, compared with melphalan. (A) Retinal function.

Electroretinography was performed weekly, 1 day prior to each of the three planned injections, as well as 1 week after the final injection (immediately prior to euthanising the rabbit). Retinal responses to scotopic 100 mcd flashes, scotopic 3000 mcd flashes, scotopic 10000 mcd flashes, photopic 3000 mcd flashes and $30 \mathrm{~Hz}$ flicker flashes were recorded. A-wave and B-wave amplitudes, and A-wave and B-wave implicit times were recorded (except for the $30 \mathrm{~Hz}$ flicker, for which there is only a B wave). Shaded areas on the graphs represent $95 \%$ Cls. No toxicity (see the Methods section for toxicity criteria) was observed for any parameter in the saline-treated control eyes, as well as in the cohorts treated with either $15 \mu \mathrm{g}$ or $30 \mu \mathrm{g}$ of topotecan. However, significant toxicity was seen in the cohort of rabbits treated with $12.5 \mu \mathrm{g}$ of melphalan. Graphs of amplitudes are shown, but similar results were seen for implicit times, as well. For those particular tests where significant toxicity was seen, per cent change and $p$ values for estimates of trend are shown alongside the particular graph. P values of the difference between groups are shown at the top of each graph. (B-F) Histopathology of treated eyes demonstrating (B) normal retinal architecture in untreated eyes and in (C) the saline-treated eyes. (D) In contrast, eyes treated with $12.5 \mu \mathrm{g}$ melphalan showed significant retinal atrophy on histopathology (arrow shows the location of loss of outer retinal architecture). Eyes treated with intravitreal injections of (E) $15 \mu \mathrm{g}$ topotecan, or (F) $30 \mu \mathrm{g}$ topotecan were histologically indistinguishable from saline-treated (or untreated) eyes. Retinal detachments are artefactual. NS=not significant. 
were 'undetectable' $(<5 \mu \mathrm{V})$, or if multiple injections occurred between the preinjection and postinjection ERGs. Thus, the final group included for analysis of toxicity and ERGs included 225 intravitreal melphalan monotherapy injections (66 at $25 \mu \mathrm{g}, 159$ at $30 \mu \mathrm{g})$.

In a univariate analysis, patients receiving melphalan experienced a $7.29 \mu \mathrm{V}$ reduction in ERG amplitude, per injection $(\mathrm{p}<0.001)$, with no significant difference in the amount of reduction between those receiving $25 \mu \mathrm{g}$ or $30 \mu \mathrm{g}$ of melphalan. In contrast, patients receiving topotecan experienced no reduction in ERG amplitude, in either the $20 \mu \mathrm{g}$ subcohort or the $30 \mu \mathrm{g}$ subcohort (figure 5).

In a mixed effect model, patients receiving melphalan experienced a $7.55 \mu \mathrm{V}$ reduction in ERG amplitude, per injection $(\mathrm{p}<0.001)$, consisting of $7.58 \mu \mathrm{V}$ reduction per $25 \mu \mathrm{g}$ injection $(\mathrm{p}=0.03)$, and $7.57 \mu \mathrm{V}$ reduction per $30 \mu \mathrm{g}$ injection $(\mathrm{p}<0.001)$. In contrast, in the mixed effect models, patients receiving topotecan at either $20 \mu \mathrm{g}$ or $30 \mu \mathrm{g}$ experienced no reduction in ERG amplitude (figure 5).

\section{Efficacy of intravitreal topotecan compared with melphalan in patients}

There were 23 patients ( 23 eyes) who were treated with intravitreal topotecan for whom a complete clinical course was available for review (follow-up: $23.5 \pm 18.8$ months). Of these six, five were treated with $30 \mu \mathrm{g}$ (all after October 2017), and one was treated with $20 \mu \mathrm{g}$. The comparator group consisted of 66 patients (70 eyes) whose entire intravitreal treatment course consisted solely of melphalan monotherapy, receiving a mean of $4.0 \pm 2.4$ injections (follow-up: $30.9 \pm 26.0$ months). In this melphalan monotherapy group, seed eradication and globe salvage was achieved in $65 / 70(92.9 \%)$ of eyes. It is difficult to evaluate the true efficacy of topotecan in this cohort as the majority of patients who received intravitreal topotecan also received intravitreal melphalan at some point during the course of treatment, and so we cannot definitively attribute the seed eradication to the topotecan in those cases. Only six patients received topotecan monotherapy exclusively throughout their intravitreal treatment course, and while the vitreous seeds were successfully eradicated in all of these patients (having received a mean of $1.8 \pm 0.75$ injections), it is possible that there might have been selection bias whereby the patients with the least significant vitreous tumour burden were most likely to receive only topotecan. Future randomised studies are needed to evaluate the relative efficacy of topotecan versus melphalan.

\section{DISCUSSION}

To assess the efficacy, toxicity and optimal therapeutic dose of intravitreal topotecan monotherapy for vitreous seeds, we performed several in vitro experiments, in vivo experiments in our rabbit model, and we report our clinical experience using intravitreal topotecan in RB patients. Our pharmacokinetic experiments and in vitro experiments calculated an optimal dose range of $15-30 \mu \mathrm{g}$ in rabbits (equivalent to $30-60 \mu \mathrm{g}$ in the larger human eye). Our in vivo efficacy experiments in our rabbit xenograft model demonstrate that $15 \mu \mathrm{g}$ topotecan is highly effective at eradicating vitreous seeds, with efficacy equivalent to standard dose melphalan. Our in vivo toxicity experiments in rabbits demonstrate that multiple injections of topotecan, even up to $30 \mu \mathrm{g}$, do not cause retinal functional or structural toxicity, in contrast to melphalan. Finally, in the clinical study, our experience with intravitreal topotecan monotherapy confirms that
$30 \mu \mathrm{g}$ in humans (equivalent to $15 \mu \mathrm{g}$ in the smaller rabbit eye) does not cause retinal toxicity in patients.

Evidence from animal models ${ }^{11} 123132$ and clinical experience, ${ }^{8} 123334$ suggests that currently used melphalan may be associated with retinal toxicity. Topotecan has been proposed as an alternative agent with efficacy against RB, and it has been incorporated into chemotherapy regimens by intravenous, ${ }^{17}$ intra-arterial ${ }^{18}$ and recently intravitreal routes. ${ }^{19}{ }^{20}$ However, topotecan has always been used in combination with other drugs for intravenous ${ }^{17} 35$ and intra-arterial regimens. ${ }^{9} 1836$ When topotecan was initially explored for intravitreal use, it was likewise combined with melphalan in an effort to increase efficacy in recalcitrant eyes, ${ }^{22}$ but Nadelmann et al have shown that combining topotecan with melphalan still causes the expected toxicity from melphalan. ${ }^{20}$ Recently, single-agent topotecan has been proposed for vitreous seeds. ${ }^{19}$ While some have reported good results, there is much variability in the doses used and many reports still ultimately include melphalan in combination, presumably because of a perceived lack of adequate efficacy at the doses selected for topotecan. A previous evaluation ${ }^{21}$ of the toxicity of intravitreal topotecan in an animal model selected a $5 \mu \mathrm{g}$ dose (equivalent to $10 \mu \mathrm{g}$ in humans), far less than the doses currently used in clinical practice, six-times less than the dose that we calculate to achieve the $\mathrm{IC}_{90}$, and three-times less than the dose we demonstrate to be clinically effective. Importantly, we demonstrate no toxicity with $15 \mu \mathrm{g}$ or even $30 \mu \mathrm{g}$ of intravitreal topotecan.

We took an evidence-based approach, rather than an exploratory trial-and-error approach, to determine the ideal dose of topotecan to study. Since each individual injection can only be given at a single location within the globe, while seeds are often diffuse throughout the vitreous cavity, the goal was to identify the concentration required at the farthest-most side of the vitreous to eradicate vitreous seeds at this farthest location. There are different factors to consider, including the rate of diffusion and the rate of efflux. The amount of drug present at the opposite side of the eye at various time-points following injection was therefore determined empirically. In vitro cytotoxicity experiments were then performed to determine the minimum concentration necessary at that location based on the pharmacokinetic parameters found in vivo, and we then calculated the initial dose that would have been required to be injected to achieve the desired concentration for a sustained length of time (five half-lives) at that farthest point. This systematic approach to dose-finding is superior to selecting several doses in a more random fashion. Since this approach identifies the required concentration at the end of five-half-lives, and at the farthest location in the eye with the lowest exposure levels, this likely represents a high-end estimate-most of the vitreous cavity is exposed to higher concentrations, and indeed at earlier time points the concentration is higher at all locations than it is at the end of the fifth half-life. In addition, this calculated effective dose assumes a single injection, whereas in practice, one would always give multiple injections. Therefore, we also explored half the calculated dose $(15 \mu \mathrm{g}$ instead of the full $30 \mu \mathrm{g}$ ). Since $15 \mu \mathrm{g}$ was shown in our rabbit model to be as effective as current melphalan doses, we then explored toxicity at the full $30 \mu \mathrm{g}$ in our rabbit model as well, and we demonstrate that there is a wide therapeutic window with topotecan.

Similarly, the ERG data of topotecan-treated patients corroborated our rabbit findings that doses of $20 \mu \mathrm{g}$ or even $30 \mu \mathrm{g}$ did not cause retinal toxicity or ERG reductions. In contrast, in our mixed effect model, melphalan caused a per-injection reduction in retinal function equivalent to $7.55 \mu \mathrm{V}$ for every 

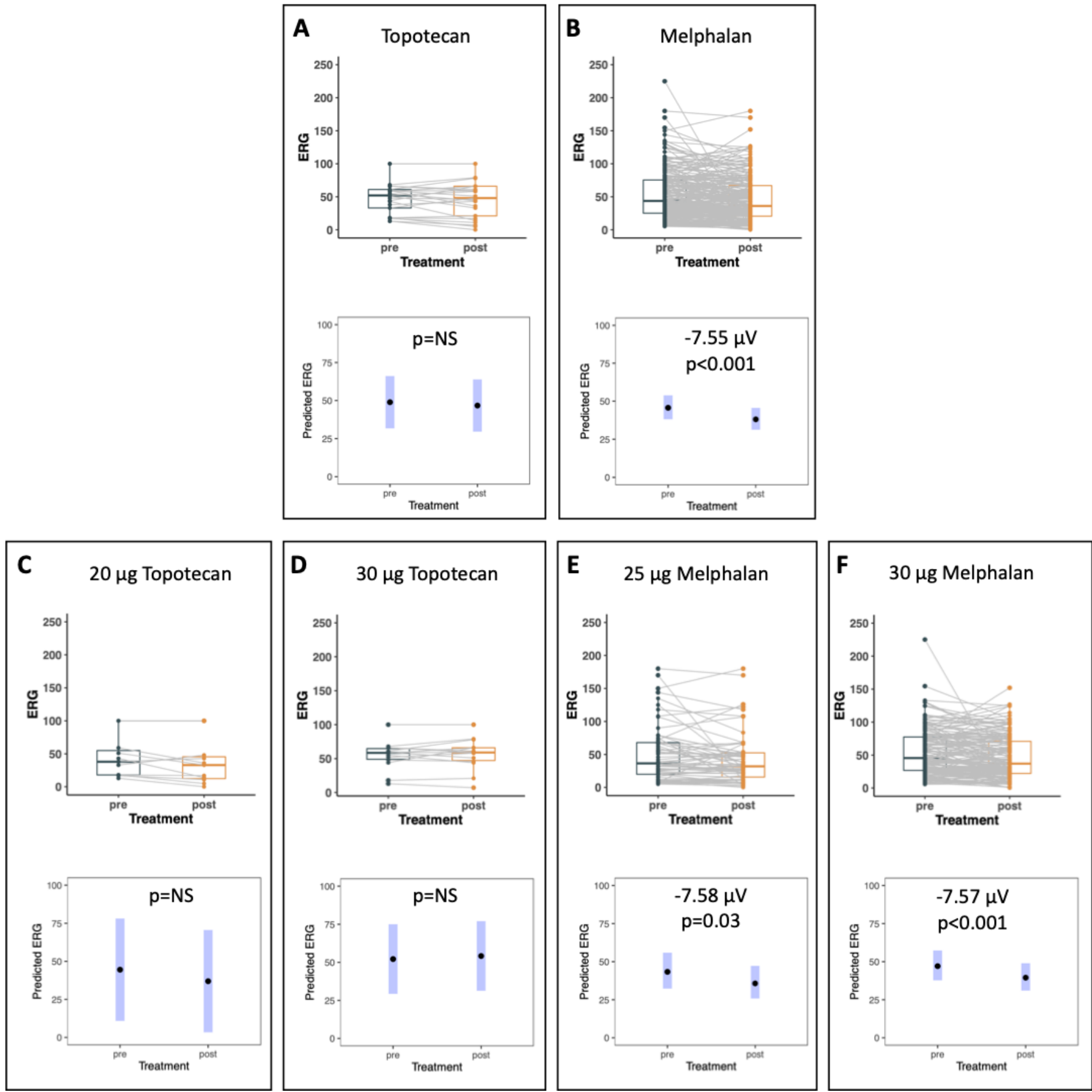

Figure 5 Changes in retinal function in topotecan-treated versus melphalan-treated eyes of patients with retinoblastoma vitreous seeds. Within each cohort or subcohort (delineated within a box), the top panel represents the univariate analysis, with each 'string' representing the electroretinography (ERG) changes with a single intravitreal injection. The bottom panel within each pair represents the results of the mixed-effect modelling, accounting for inter-eye/intra-patient and intra-eye correlations, with the appropriate statistical analysis results labelled on the panel. (A-B) ERG amplitude changes per injection for patients treated with topotecan (A), or melphalan (B). Topotecan caused no significant reduction in ERG parameters, whereas significant reductions in ERG amplitudes were seen with each injection of melphalan $(7.55 \mu \mathrm{V}$ per injection, $\mathrm{p}<0.001)$. (C-F) ERG amplitude changes by drug and dose. (C-D) represent sub-cohorts of the full topotecan-treated cohort presented in (A), and (E-F) represent subcohorts of the full melphalan-treated cohort presented in (B). Topotecan caused no reduction in ERG parameters at either $20 \mu \mathrm{g}(\mathrm{C})$ or $30 \mu \mathrm{g}(\mathrm{D})$, whereas significant reductions in ERG amplitudes were seen with each injection of either $25 \mu \mathrm{g}$ melphalan $(\mathrm{E} ; 7.58 \mu \mathrm{V}$ per injection, $\mathrm{p}=0.03)$ or $30 \mu \mathrm{g}$ melphalan ( $F ; 7.57 \mu \mathrm{V}$ per injection, $\mathrm{p}<0.001)$. NS=not significant.

melphalan injection, consistent with previous publications by our group. ${ }^{12} 3738$ There are two commonly used formulations of melphalan: (traditional) melphalan hydrochloride and captisol-stabilised propylene glycol-free melphalan. In the rabbit experiments, all rabbits were treated with traditional melphalan hydrochloride. In the patient cohort, patients treated up until 2015 (at VUMC) and up until 2016 (at MSKCC) were treated with melphalan hydrochloride, while all patients treated after those dates were treated with the newer propylene glycol-free formulation. We have previously shown that the efficacy and the 
toxicity of both formulations do not differ. ${ }^{38}$ However, it should be pointed out that not all eyes receiving melphalan will necessarily experience worsened visual function. As seen in figure 5, while there was a reduction in ERG amplitudes on average, some eyes experienced little or no ERG reductions. It should also be pointed out that macular toxicity (including cystoid macular oedema), which has been reported to occur occasionally with intravitreal injections, ${ }^{39}$ might not result in measurable reduction in retina-wide, full-field ERG. The specific factors influencing retinal toxicity in a given patient are not clear.

\section{CONCLUSIONS}

Taken together, our preclinical and clinical findings support that topotecan $30 \mu \mathrm{g}$ (equivalent to $15 \mu \mathrm{g}$ in our rabbit models) appears to cause no retinal toxicity in the rabbit model or in patients. Our rabbit model data indicate that topotecan might be equally effective to melphalan, supporting the need for future clinical studies that directly compare the efficacy in patients.

\author{
Author affiliations \\ ${ }^{1}$ Ophthalmology \& Visual Sciences, Vanderbilt University Medical Center, Nashville, \\ Tennessee, USA \\ ${ }^{2}$ Center for Quantitative Sciences, Vanderbilt University Medical Center, Nashville, \\ Tennessee, USA \\ ${ }^{3}$ Vanderbilt-Ingram Cancer Center, Vanderbilt University Medical Center, Nashville, \\ Tennessee, USA \\ ${ }^{4}$ Pathology, Microbiology and Immunology, Vanderbilt University Medical Center, \\ Nashville, Tennessee, USA \\ ${ }^{5}$ Biochemistry, Vanderbilt University, Nashville, Tennessee, USA \\ ${ }^{6}$ Center for Neuroscience Drug Discovery, Vanderbilt University, Nashville, Tennessee, \\ USA \\ ${ }^{7}$ Surgery, Memorial Sloan Kettering Cancer Center, New York, New York, USA \\ ${ }^{8}$ Pediatrics, Vanderbilt University Medical Center, Nashville, Tennessee, USA \\ ${ }^{9}$ Pharmacology, Vanderbilt University, Nashville, Tennessee, USA \\ ${ }^{10}$ VA Tennessee Valley Healthcare System Nashville Campus, Nashville, Tennessee, \\ USA \\ ${ }^{11}$ Program in Cancer Biology, Vanderbilt University, Nashville, Tennessee, USA \\ ${ }^{12}$ Radiation Oncology, Vanderbilt University Medical Center, Nashville, Tennessee, \\ USA
}

\section{Twitter Jessica V Kaczmarek @KaczmarekMD and Anthony B Daniels @ AnthonyBDaniels}

Contributors Designed the study: JMP, DLF, AR, ABD. Performed the experiments: CMB, JVK, JMP, KLB, MWC, DHA, JHF, ABD. Analysed the data: CMB, JVK, JMP, S-CC, $\mathrm{KLB}, \mathrm{MWC}, \mathrm{TMB}, \mathrm{CWL}, \mathrm{JN}, \mathrm{AL}, \mathrm{TH}, \mathrm{DHA}, J H F, \mathrm{DLF}, \mathrm{AR}, \mathrm{ABD}$. Prepared the manuscript: $D L F, A R, A B D$.

Funding This work was supported by a Career Development Award from the Research to Prevent Blindness Foundation (ABD) (no specific grant number), by the National Eye Institute grant NIH/NEI K08EY027464 (ABD), by an unrestricted departmental grant from Research to Prevent Blindness to the Vanderbilt Department of Ophthalmology and Visual Sciences (no specific grant number), by a Department of Veterans Affairs Senior Research Career Scientist Award IK6BX005225 (AR), and the Vanderbilt Ingram Cancer Center Support Grant (P30 CA68485) and the Memorial Sloan-Kettering Cancer Center Support Grant (P30 CA008748) for core facilities. It was also supported by P41 GM103391 and by the National Center for Research Resources, Grant UL1RR024975, now at the National Center for Advancing Translational Sciences (2 UL1 TR000445). The content is the sole responsibility of the authors and does not necessarily represent the official views of the NIH.

Competing interests $A B D$ and DLF have a patent with Vanderbilt University Medical Center. ABD has received research funding from Spectrum Pharmaceuticals (now Acrotech Biopharma) through an investigator-initiated study separate from the data presented in this manuscript. None of the other authors has any conflicts of interest or financial disclosures.

\section{Patient consent for publication Not required}

Ethics approval The Institutional Review Board approval was obtained at both Vanderbilt University Medical Center and Memorial Sloan-Kettering Cancer Center. Informed consent was obtained from patients for all procedures performed. This study adhered to the tenets of the Declaration of Helsinki and was performed in accordance with the Health Insurance Portability and Accountability Act. Data are available from the investigators upon request.
Provenance and peer review Not commissioned; externally peer reviewed.

Data availability statement Data are available upon reasonable request. For laboratory data, contact anthony.b.daniels@vumc.org. For deidentified patient data, contact anthony.b.daniels@vumc.org and abramsod@mskcc.org.

Open access This is an open access article distributed in accordance with the Creative Commons Attribution Non Commercial (CC BY-NC 4.0) license, which permits others to distribute, remix, adapt, build upon this work non-commercially, and license their derivative works on different terms, provided the original work is properly cited, appropriate credit is given, any changes made indicated, and the use is non-commercial. See: http://creativecommons.org/licenses/by-nc/4.0/.

\section{ORCID iDs}

Jennifer B Nadelmann http://orcid.org/0000-0002-7021-9986

Jasmine H Francis http://orcid.org/0000-0002-1889-3447

Anthony B Daniels http://orcid.org/0000-0001-8361-9885

\section{REFERENCES}

1 Shields CL, Mashayekhi A, Au AK, et al. The International classification of retinoblastoma predicts chemoreduction success. Ophthalmology 2006;113:2276-80.

2 Shields CL, Honavar SG, Meadows AT, et al. Chemoreduction plus focal therapy for retinoblastoma: factors predictive of need for treatment with external beam radiotherapy or enucleation. Am J Ophthalmol 2002;133:657-64.

3 Abramson DH, Beaverson KL, Chang ST, et al. Outcome following initial external beam radiotherapy in patients with Reese-Ellsworth group Vb retinoblastoma. Arch Ophthalmol 2004;122:1316-23.

4 Murphree AL, Villablanca JG, Deegan WF, et al. Chemotherapy plus local treatment in the management of intraocular retinoblastoma. Arch Ophthalmol 1996;114:1348-56.

5 Daniels AB, Patel SN, Milam RW, et al. Effect of intravenous chemotherapy regimen on globe salvage success rates for retinoblastoma based on disease Class-A metaanalysis. Cancers 2021;13:2216.

6 Abramson DH, Dunkel IJ, Brodie SE, et al. A phase I/II study of direct intraarterial (ophthalmic artery) chemotherapy with melphalan for intraocular retinoblastoma initial results. Ophthalmology 2008;115:1404 e1391:1398-404.

7 Munier FL, Soliman S, Moulin AP, et al. Profiling safety of intravitreal injections for retinoblastoma using an anti-reflux procedure and sterilisation of the needle track. $\mathrm{Br}$ J Ophthalmol 2012;96:1084-7

8 Munier FL, Gaillard M-C, Balmer A, et al. Intravitreal chemotherapy for vitreous disease in retinoblastoma revisited: from prohibition to conditional indications. $\mathrm{Br} J$ Ophthalmol 2012:96:1078-83.

9 Abramson DH, Daniels AB, Marr BP, et al. Intra-Arterial chemotherapy (ophthalmic artery Chemosurgery) for group D retinoblastoma. PLoS One 2016;11:e0146582.

10 Berry JL, Shah S, Bechtold M, et al. Long-Term outcomes of group D retinoblastoma eyes during the intravitreal melphalan era. Pediatr Blood Cancer 2017;64. doi:10.1002/pbc.26696. [Epub ahead of print: 2406 2017].

11 Daniels AB, Froehler MT, Nunnally AH, et al. Rabbit model of intra-arterial chemotherapy toxicity demonstrates retinopathy and vasculopathy related to drug and dose, not procedure or approach. Invest Ophthalmol Vis Sci 2019;60:954-64.

12 Francis JH, Schaiquevich P, Buitrago E, et al. Local and systemic toxicity of intravitreal melphalan for vitreous seeding in retinoblastoma: a preclinical and clinical study. Ophthalmology 2014:121:1810-7.

13 Xue K, Ren H, Meng F, et al. Ocular toxicity of intravitreal melphalan for retinoblastoma in Chinese patients. BMC Ophthalmol 2019;19:61.

14 Hsieh T, Liao A, Francis JH, et al. Comparison of efficacy and toxicity of intravitreal melphalan formulations for retinoblastoma. PLoS One 2020;15:e0235016.

15 Liao A, Hsieh T, Francis JH, et al. Toxicity and efficacy of intravitreal melphalan for retinoblastoma: $25 \mu \mathrm{g}$ versus $30 \mu \mathrm{g}$. Retina 2021;41:208-12.

16 Smith SJ, Smith BD, Mohney BG. Ocular side effects following intravitreal injection therapy for retinoblastoma: a systematic review. Br J Ophthalmol 2014;98:292-7.

17 Qaddoumi I, Billups CA, Tagen $M$, et al. Topotecan and vincristine combination is effective against advanced bilateral intraocular retinoblastoma and has manageable toxicity. Cancer 2012;118:5663-70.

18 Marr BP, Brodie SE, Dunkel IJ, et al. Three-Drug intra-arterial chemotherapy using simultaneous carboplatin, topotecan and melphalan for intraocular retinoblastoma: preliminary results. Br J Ophthalmol 2012;96:1300-3.

19 Rao R, Honavar SG, Sharma V, et al. Intravitreal topotecan in the management of refractory and recurrent vitreous seeds in retinoblastoma. Br J Ophthalmol 2018;102:490-5

20 Nadelmann J, Francis JH, Brodie SE, et al. Is intravitreal topotecan toxic to retinal function? Br J Ophthalmol 2020. doi:10.1136/bjophthalmol-2020-316588. [Epub ahead of print: 14 Jul 2020].

21 Buitrago E, Del Sole MJ, Torbidoni A, et al. Ocular and systemic toxicity of intravitreal topotecan in rabbits for potential treatment of retinoblastoma. Exp Eye Res 2013;108:103-9.

22 Ghassemi F, Shields CL, Ghadimi H, et al. Combined intravitreal melphalan and topotecan for refractory or recurrent vitreous seeding from retinoblastoma. JAMA Ophthalmol 2014;132:936-41. 
23 Daniels AB, Froehler MT, Pierce JM, et al. Pharmacokinetics, tissue localization, toxicity, and treatment efficacy in the first small animal (rabbit) model of intra-arterial chemotherapy for retinoblastoma. Invest Ophthalmol Vis Sci 2018;59:446-54.

24 Oatess TL, Chen PH, Daniels AB, et al. Severe Periocular Edema after Intraarterial Carboplatin Chemotherapy for Retinoblastoma in a Rabbit (Oryctolagus cuniculus) Model. Comp Med 2020;70:176-82.

25 Daniels AB, Pierce JM, Chen S-c. Complete preclinical platform for intravitreal chemotherapy drug discovery for retinoblastoma: assessment of pharmacokinetics, toxicity and efficacy using a rabbit model. MethodsX 2021;113:101358.

26 Berry JL, Bechtold M, Shah S, et al. Not All Seeds Are Created Equal: Seed Classification Is Predictive of Outcomes in Retinoblastoma. Ophthalmology 2017; 124:1817-25.

27 Gjörloff K, Andréasson S, Ehinger B. Standardized full-field electroretinography in rabbits. Doc Ophthalmol 2004;109:163-8.

28 Liu CY, Jonna G, Francis JH, et al. Non-selectivity of ERG reductions in eyes treated for retinoblastoma. Doc Ophthalmol 2014;128:13-23.

29 Brodie SE, Munier FL, Francis JH, et al. Persistence of retinal function after intravitreal melphalan injection for retinoblastoma. Doc Ophthalmol 2013;126:79-84.

30 Brodie SE, Pierre Gobin Y, Dunkel IJ, et al. Persistence of retinal function after selective ophthalmic artery chemotherapy infusion for retinoblastoma. Doc Ophthalmol 2009;119:13-22.

31 Tse BC, Steinle JJ, Johnson D, et al. Superselective intraophthalmic artery chemotherapy in a nonhuman primate model: histopathologic findings. JAMA Ophthalmol 2013;131:903-11.
32 Steinle JJ, Zhang Q, Thompson KE, et al. Intra-ophthalmic artery chemotherapy triggers vascular toxicity through endothelial cell inflammation and leukostasis. Invest Ophthalmol Vis Sci 2012;53:2439-45.

33 Ghassemi F, Amoli FA. Pathological findings in enucleated eyes after intravitreal melphalan injection. Int Ophthalmol 2014;34:533-40.

34 Aziz HA, Kim JW, Munier FL, et al. Acute hemorrhagic retinopathy following intravitreal melphalan injection for retinoblastoma: a report of two cases and technical modifications to enhance the prevention of retinal toxicity. Ocul Oncol Pathol 2017:3:34-40.

35 Brennan RC, Qaddoumi I, Mao S, et al. Ocular salvage and vision preservation using a Topotecan-Based regimen for advanced intraocular retinoblastoma. JCO 2017;35:72-7

36 Francis JH, Gobin YP, Dunkel IJ, et al. Carboplatin +/- topotecan ophthalmic artery chemosurgery for intraocular retinoblastoma. PLoS One 2013;8:e72441.

37 Francis JH, Brodie SE, Marr B, et al. Efficacy and toxicity of Intravitreous chemotherapy for retinoblastoma: four-year experience. Ophthalmology 2017;124:488-95.

38 Bogan CM, Pierce JM, Doss SD, et al. Intravitreal melphalan hydrochloride vs propylene glycol-free melphalan for retinoblastoma vitreous seeds: efficacy, toxicity and stability in rabbits models and patients. Exp Eye Res 2021;204:108439.

39 Panthagani J, Montecinos P, López JP, et al. Cystoid macular edema following intravitreal chemotherapy treatment for retinoblastoma. Pediatr Blood Cancer 2020;67:e28348. 